\title{
549.
}

\section{NOTE ON THE MAXIMA OF CERTAIN FACTORIAL FUNCTIONS.}

[From the Messenger of Mathematics, vol. II. (1873), pp. 129, 130.]

I CONSIDER the functions

$$
\begin{aligned}
& y_{1}=x(x-1) \\
& y_{2}=x\left(x-\frac{1}{2}\right)(x-1) \\
& y_{3}=x\left(x-\frac{1}{3}\right)\left(x-\frac{2}{3}\right)(x-1), \\
& \vdots \\
& y_{n}=x\left(x-\frac{1}{n}\right)\left(x-\frac{2}{n}\right) \ldots\left(x-\frac{n-1}{n}\right)(x-1) .
\end{aligned}
$$

Attending only to the absolute values, disregarding the signs, $y_{n}$ has $n$ maxima, viz. if $n$ be odd, $=2 p+1$ suppose, these are

$$
Y_{1}, Y_{2}, \ldots, Y_{p}, Y_{p+1}, Y_{p}, \ldots Y_{1}
$$

where $Y_{p+1}$ corresponds to the value $x=\frac{1}{2}$, and $Y_{1}, Y_{2}, \ldots, Y_{p}$ to values of $x$ between

$$
0 \text { and } \frac{1}{2 p+1}, \frac{1}{2 p+1} \text { and } \frac{2}{2 p+1}, \ldots, \frac{p-1}{2 p+1} \text { and } \frac{p}{2 p+1} \text {. }
$$

But if $n$ be even, $=2 p$ suppose, then the maxima are

$$
Y_{1}, Y_{2}, \ldots, Y_{p}, Y_{p}, \ldots, Y_{1},
$$

where $Y_{1}, Y_{2}, \ldots, Y_{p}$ correspond to values of $x$ between

$$
0 \text { and } \frac{1}{2 p}, \frac{1}{2 p} \text { and } \frac{2}{2 p}, \ldots, \frac{p-1}{2 p} \text { and } \frac{1}{2} \text {. }
$$


In every case the maxima decrease from $Y_{1}$ which is the greatest, to $Y_{p}$ or $Y_{p+1}$ which is the least; in particular, $n=2 p+1$, then

which is

$$
\begin{aligned}
Y_{p+1} & =\frac{1}{2}\left(\frac{1}{2}-\frac{1}{2 p+1}\right) \cdots\left(\frac{1}{2}-1\right) \\
& =\left(\frac{1}{2} \frac{2 p-1}{2.2 p+1} \cdots \frac{1}{2.2 p+1}\right)^{2} \\
& =\frac{\{1.3 \ldots(2 p-1)\}^{2}}{2^{2 p+2} \cdot(2 p+1)^{2 p}}=\frac{1}{4} \frac{\left(\frac{1}{2} \cdot \frac{3}{2} \cdots p-\frac{1}{2}\right)^{2}}{(2 p+1)^{2 p}}, \\
& =\frac{1}{4} \frac{\left\{\Gamma\left(p+\frac{1}{2}\right) \div \Gamma \frac{1}{2}\right\}^{2}}{(2 p+1)^{2 p}}=\frac{\Gamma^{2}\left(p+\frac{1}{2}\right)}{4 \pi(2 p+1)^{2 p}} .
\end{aligned}
$$

Suppose $p$ is large; then, as for large values of $x$,

we have

$$
\Gamma x=\sqrt{ }(2 \pi) x^{x-\frac{1}{2}} e^{-x},
$$

$$
\begin{aligned}
\Gamma\left(p+\frac{1}{2}\right) & =\sqrt{ }(2 \pi)\left(p+\frac{1}{2}\right)^{p} e^{-p-\frac{1}{2}} \\
& =\sqrt{ }(2 \pi) p^{p} e^{p \log \left(1+\frac{1}{2 p}\right)} e^{-p-\frac{1}{2}}=\sqrt{ }(2 \pi) p^{p} e^{-p}, \\
(2 p+1)^{2 p} & =(2 p)^{2 p} \cdot e^{2 p \log \left(1+\frac{1}{2 p}\right)}=2^{2 p} p^{2 p} e
\end{aligned}
$$

and so

$$
Y_{p+1}=\frac{2 \pi p^{2 p} e^{-2 p}}{4 \pi 2^{2 p} p^{2 p} e}=\frac{p^{2} e^{-2 p-1}}{2^{2 p-1} \cdot}=p^{2}\left(\frac{1}{2 e}\right)^{2 p+1} .
$$

Also $Y_{1}$ corresponds approximately to

$$
\begin{gathered}
x=\frac{1}{2} \frac{1}{2 p+1}=\frac{1}{2 n} \\
Y_{1}=\frac{1}{2 n} \cdot \frac{1}{2 n} \cdot \frac{3}{2 n} \ldots \frac{2 n-1}{2 n}=\frac{1}{n^{n+1} \frac{1}{2} \cdot \frac{1}{2} \cdot \frac{3}{2} \ldots\left(n-\frac{1}{2}\right)} \\
=\frac{1}{n^{n+1} \frac{1}{2}} \Gamma \frac{\Gamma\left(n+\frac{1}{2}\right)}{\Gamma \frac{1}{2}}=\frac{1}{2(2 p+1)^{2 p+1}} \frac{\Gamma\left(2 p+\frac{3}{2}\right)}{\sqrt{ }(\pi)} .
\end{gathered}
$$

Now$$
\Gamma\left(2 p+\frac{3}{2}\right)=\sqrt{ }(2 \pi)\left(2 p+\frac{3}{2}\right) e^{-2 p-\frac{3}{2}}=\sqrt{ }(2 \pi)(2 p)^{2 p+2} e^{\left(2 p+\frac{3}{2}\right) \log \left(1+\frac{3}{4 p}\right)} e^{-2 p-\frac{3}{2}}
$$$$
=\sqrt{ }(2 \pi) 2^{2 p+2} p^{2 p+2} e^{-2 p},
$$

and

so that

$$
(2 p+1)^{2 p+1}=(2 p)^{2 p+1} e^{(2 p+1) \log \left(1+\frac{1}{2 p}\right)}=(2 p)^{2 p+1} e ;
$$

$$
\begin{aligned}
Y_{1} & =\frac{1}{2^{2 p+2} p^{2 p+1} e} \cdot \frac{\sqrt{ }(2 \pi) \cdot 2^{2 p+2} \cdot p^{2 p+2} e^{-2 p}}{\sqrt{ }(\pi)} \\
& =\frac{p \sqrt{ }(2)}{e^{2 p+1}}
\end{aligned}
$$

so that, $p$ being large, $Y_{1}$ is far larger than $Y_{p+1}$. 